\title{
SmartAgeing: a 3D serious game for early detection of mild cognitive impairments
}

\author{
Dani Tost \\ CREB-UPC \\ dani@lsi.upc.edu \\ Stefania Pazzi \\ CBIM \\ s.pazzi@cbim.it
}

\author{
Ariel von Barnekow \\ CREB-UPC \\ Stefano Puricelli
CBIM
s.puricelli@cbim.it
}

ariel.von.barnekow@upc.edu

\author{
Sara Bottiroli \\ National Neurological Institute \\ C. Mondino \\ sara.bottiroli@unipv.it
}

\begin{abstract}
We describe SmartAgeing, a serious game in a 3D virtual environment aimed at the early detection of Mild Cognitive Impairments in persons ageing between 50 and 80, and at assessing cognitive impairments in persons already diagnosed or having neurodegenerative dementia. SmartAgeing is a telematic system in which users realize a set of screening tests structured in five daily-life tasks in a familiar environment addressing various cognitive skills: memory, executive functions, divided attention, short-term and longterm memory and spatial orientation and attention. The game integrates novel accessibility features to make it usable by seniors. Several indexes of evaluation are registered while users perform the test, that provide a complete picture of the user's behavior during the game. The design and implementation of the game has been realized following a user-centered agile methodology that has allowed to refine the tasks and enhance the technology throughout the development stage. The game is currently in a validation stage on a sample of 1000 users. The results of the electronic test are been compared to standard pen-based tests. We except that the validation will confirm SmartAgeing as a powerful screening tool for the early detection of cognitive impairments on a wide scale, otherwise impossible using the traditional pen-based test.
\end{abstract}

\section{Categories and Subject Descriptors}

I.3.7 [Computer Graphics]: Three Dimensional Graphics and Realism, Animation, Virtual Reality; J.3 [Computer Applications]: Life and Medical Sciences; H.5.2 [Information Interfaces and Presentation]: User Interfaces.

Permission to make digital or hard copies of all or part of this work for personal or classroom use is granted without fee provided that copies are not made or distributed for profit or commercial advantage and that copies bear this notice and the full citation on the first page. To copy otherwise, to republish, to post on servers or to redistribute to lists, requires prior specific permission and/or a fee. REHAB 2014, May 20-23, Oldenburg, Germany Copyright @ 2014 ICST 978-1-63190-011-2 DOI 10.4108/icst.pervasivehealth.2014.255334

\section{Keywords}

Serious Games, Mild Cognitive Impairments, Ageing, Screening.

\section{INTRODUCTION}

There is a general agreement on the idea that playing can boost brain functions and improve well-being [2]. Playing is a pervasive cognitively demanding activity that, as shown in a recent study [7], can yield to structural changes in the brain. Serious Games (SG) designed on a hand-to-hand collaboration between game developers and neuroscientists can have a more effective impact on cognitive training than purely leisure games [1].

In particular, SG potentially represent new and effective tools in the management and treatment of cognitive impairments in the elderly [3]. Indeed, there are evidences that adults engaged in computer activities have decreased odds of developing MCI [9] and that virtual reality-based memory training can contribute to prevent memory decline [10] and reduce depressive symptoms [6] in elderly adults.

Our hypothesis is that in addition to rehabilitation and training, SG can also be an effective tool for the detection of cognitive impairments and dementia. Therefore, they can be used to perform large-scale, cheaper screening of the population over 50 yielding to earlier detection of cognitive impairments and anticipated enrollment in rehabilitation programs.

On the basis of this hypothesis and with the ambition of partially substituting pen-and-paper-based tests, we have designed and implemented SmartAgeing, a web-based electronic test of Mild Cognitive Impairments (MCI) based on the SG technology. In the development of this new technology, we have faced various challenges to make it accessible to a diversity of users most of them without computer skills. We describe our solution in the next sections.

\section{SCENARIO}

The scenario represents a 3D home-like space (loft) providing a diversity of ambiances in one room: dining room, sitting room, bedroom and kitchen, plus a separate bathroom. 


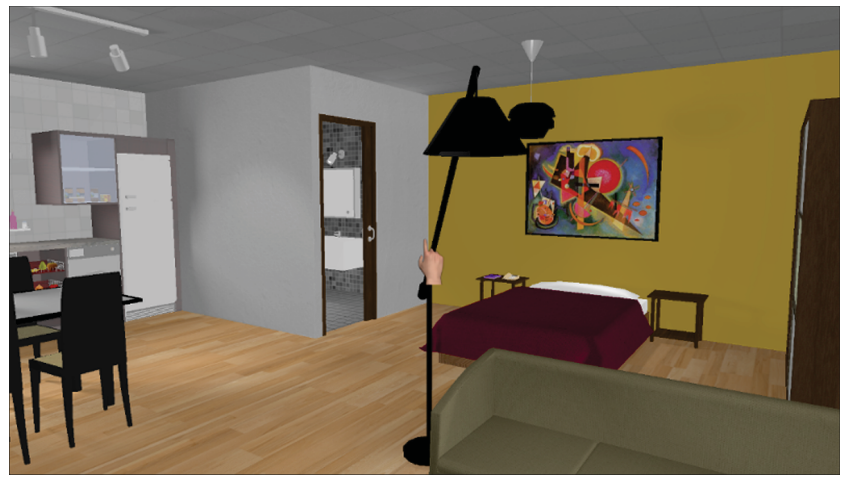

Figure 1: A view of the virtual scenario

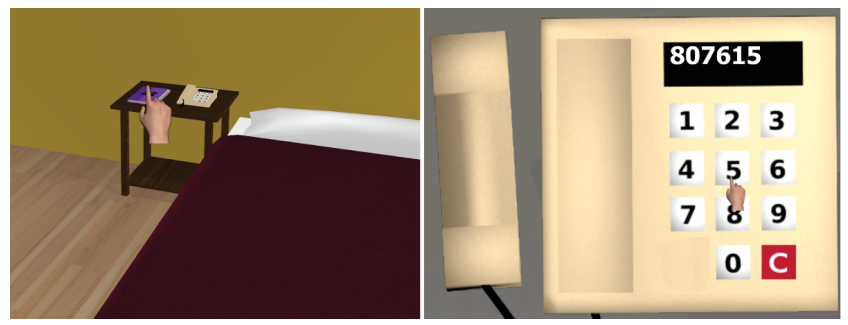

Figure 2: An example of integration of 2D tasks in the 3D environment

It is filled with furniture and objects that users can manipulate virtually. This scenario provides means of developing very varied exercises within the same space (see Figure 2).

The game is designed in a first-person perspective so the player experiences the action through the eyes of an invisible avatar. The player walks virtually in the environment and interacts with the objects using a touch interface in order to fulfill the tasks following written instructions that appear in the bottom of the screen and in a side pop-up panel.

The actions are dimensionally congruent [4]: those related with objects' manipulation are done in $3 \mathrm{D}$, but others that are inherently $2 \mathrm{D}$ such as reading and writing are done using $2 \mathrm{D}$ interfaces launched from the 3D virtual environment. As an example, in order to dial a telephone number, users interact with the 3D model of the telephone. This causes the display of a $2 \mathrm{D}$ view of the telephone where users can dial the numbers very easily (see Figure 2). Once finished, the game comes back to the last view of the $3 \mathrm{D}$ scenario. In this way, the game naturally accommodates the two visualization and interaction models within a same framework.

\section{TASKS}

The test consists of five tasks addressing different cognitive functions: executive functions (reasoning and planning), attention (selected and divided), memory (short and long term, perspective), orientation (visuo-spatial). Table 5 summarizes the tasks. The game starts with a traveling in the environment to familiarize the user with it. During the traveling, all the doors and drawers of the cupboards and furniture of the loft are opened to show their content. Then, there is a task of familiarization in which users practice the navi-

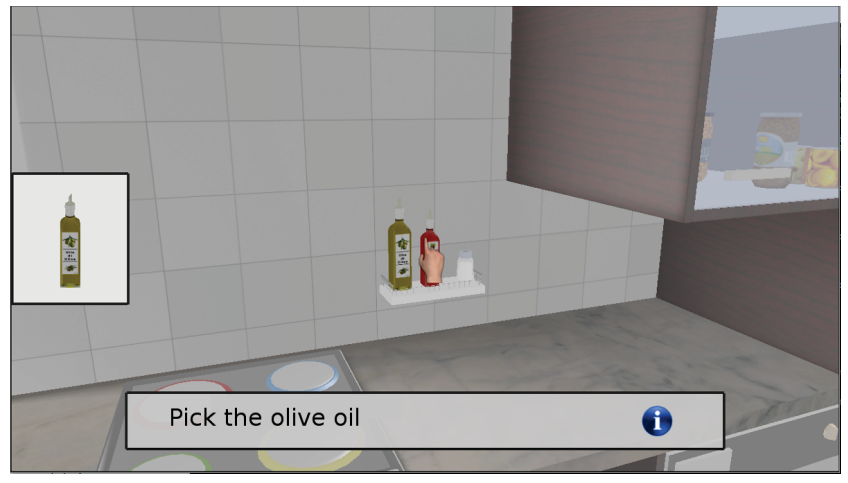

Figure 3: A view of task 1

gation and the selection of objects. After that, the first task consists of finding in the environment the objects shown in a panel at the left of the screen (see Figure 3). The image is the panel is a representative view of the objects from which they are clearly identifiable. The location of the objects in the scenario is logical: there are placed at usual locations in the real life. All the objects have been shown in their current location in the initial traveling. The task requires memory, spatial orientation and attention at the same time.

In the second task users are asked to turn on the radio and listen. They must click a button each time they listen a given word. After a while, they are required to continue confirming that they have listened the word, but at the same time to water the flowers. To do so, they must realize a sequential activity: pick the watering can, fill it in the tap (which requires to first turn on the faucet and after having filled, recalling to turn it off), and finally, watering the flowers. Thus, the task involves divided attention and executive planning functions.

In the third task users are first warned to turn on the television at the end of the task. Then, they must select the agenda that is on top of the table near the bed. The selection deploys a 2D interface where users must find the telephone number of a given person. Then the telephone number is shown and users learn it during a lapse of time. After that, the game comes back to the $3 \mathrm{D}$ environment where users select the telephone which, as mentioned before, results on the deployment of a $2 \mathrm{D}$ interface for dialing (short-term-memory). At the end of the task users must remember to turn off the television (long-term memory). This task involves memory and executive functions and selective attention.

Finally tasks 4 and 5 are related to task 1 . In task 4 , the user must identify the objects selected in task 1 from a $2 \mathrm{D}$ panel. This task tests memory. In task 5 the user must re-do the identification of objects in 3D as in task 1, but without following instructions and without order. It is therefore a long-term memory exercise coupled with spatial orientation and attention.

\section{USER-FRIENDLY INTERFACE}

The interface has been specially designed to make it accessible to older or non-expert users. Navigation in 3D is 
Table 1: Tasks of the test

\begin{tabular}{|c|c|}
\hline Description & Cognitive function \\
\hline $\begin{array}{c}\text { Task 1: Identification } \\
\text { Identify the objects shown } \\
\text { in the panel within } \\
\text { the kitchen }\end{array}$ & $\begin{array}{c}\text { Memory } \\
\text { Spatial orientation } \\
\text { Attention }\end{array}$ \\
\hline $\begin{array}{c}\text { Task 2: Water the flowers } \\
\text { Turn on the radio. } \\
\text { Press a button each time } \\
\text { you listen a specific word } \\
\text { Meanwhile, water the flowers }\end{array}$ & $\begin{array}{l}\text { Executive functions } \\
\text { (planning) } \\
\text { Divided attention }\end{array}$ \\
\hline $\begin{array}{l}\text { Task 3: Phone call } \\
\text { Look for the telephone of } \\
\text { of Mr. X in the phone book } \\
\text { Memorize the number and call. } \\
\text { At the end, turn-on the TV. }\end{array}$ & $\begin{array}{l}\text { Executive functions } \\
\text { Selective attention } \\
\text { Short-term and } \\
\text { long-term memory }\end{array}$ \\
\hline $\begin{array}{c}\text { Task 4: Selection } \\
\text { Click on the } 12 \text { objects } \\
\text { identified in the first task } \\
\text { from a a } 2 \mathrm{D} \text { panel with } 12 \\
\text { impostors }\end{array}$ & Memory \\
\hline $\begin{array}{c}\text { Task 5: Identification } \\
\text { Find the objects identified } \\
\text { in the first task without } \\
\text { a help panel }\end{array}$ & $\begin{array}{l}\text { Long-term memory } \\
\text { Spatial orientation } \\
\text { Attention }\end{array}$ \\
\hline
\end{tabular}

probably the more difficult manipulation for users with no experience in 3D gaming. To make the task accessible for senior users, we have implemented an automatic navigation system [8]. Users just need to click on the location where then want to go. The system computes automatically the best path to reach this location and follows this path, by simulating a translation on the floor's plane at a constant height. In this way, users have all the advantages of a 3D environment, but they are not required to master locomotion. To orient the camera at a still position they use a two degrees-of-freedom interface allowing them to fix the pitch and yaw angles (elevation and axial rotation of the head). To prevent exaggerate elevations, a maximum pitch angle is fixed. When the user is still, if the view orientation is not parallel to floor plane, the system smoothly recovers the horizontality.

In the first version of the game, the camera orientation was done by mapping the mouse movement to pitch and yaw rotations and the selection corresponded to clicks on the left mouse button. However, the preliminary user tests showed that elderly users had difficulties in managing the mouse. Therefore, we have implemented a touch-screen version of the interface. The $3 \mathrm{D}$ view is surrounded by a $2 \mathrm{D}$ frame. Users touch the upper frame to elevate the head (pitch angle) and the bottom frame to lower it. They touch the left and right frame to rotate it (yaw angle). When needed for the task, the frame incorporates a button image. In the 3D view, users select an object by touching it.

\section{INDICES AND VALIDATION}

The game records all users actions. From these data, it computes a set of indices for each task of the game separately. At the end of the game these indices are parsed to give an overall score of the patient's cognitive skills. Currently, clin-
Table 2: Main indices registered for each task of the game

\begin{tabular}{|c|}
\hline Interactions \\
\hline Number of navigation interactions \\
Number of interactions on 3D objects \\
Number of interactions on 2D objects \\
Number of interactions on 2D widgets (button) \\
\hline Actions \\
\hline Number of correct actions on time \\
Number of correct actions out-of-time \\
Number of incorrect actions \\
\hline Navigation \\
\hline Distance travel led \\
Time \\
Total \\
Per action \\
Void \\
Between interactions
\end{tabular}

icians are working on the evaluation model, with the goal of making it equivalent to standardized measures based on pen and paper screening tests. Recording all the indices provides flexibility in adjusting the model during the validation stage.

The parameters registered by the game are summarized in Table 5. We differentiate between correct and incorrect actions. Correct actions can be done on time or within a predefined delay. For instance, in task 1, identifying an object is correct and on time if the picture of the object is shown in the panel when the identification is done. It is correct but not in time when it is identified after the object has been shown and within a given delay. It is incorrect if the object has never been shown before or when the delay has expired.

\section{VALIDATION}

The validation stage has just started with a primary target group represented by 1000,50 years old and older persons, 50 to 80 of which are already diagnosed with MCI and/or neurodegenerative dementia. MCI patients will be recruited from two neurological institutes: IRCCS Mondino and Don Gnocchi Foundation, which are two important centers for the diagnosis and treatment of neurological disorders. Subjects will be already diagnosed as affected by MCI according to [11] criteria and selected from the institutions' patient registries. The inclusion criteria are a Mini Mental State Examination - MMSE [5] score $>24$ and the memory domain subscale of the Clinical Dementia Rating Scale $<0.5$.

The sample of 1000 users will be recruited from aggregation centers, public entities as well as through newspaper advertisements. The sample will be stratified according to gender (female/male), age (50-60, 61-70, 71-80), and education (primary, middle, high, university). The inclusion criteria are a negative history for neurologic and psychiatric diseases, a MMSE score $>24$ and a performance within normative ranges for age and education at the pen and pencil tests. It is expected that at least $8 \%$ [12] of these participants will be excluded from the validation stage failing to 


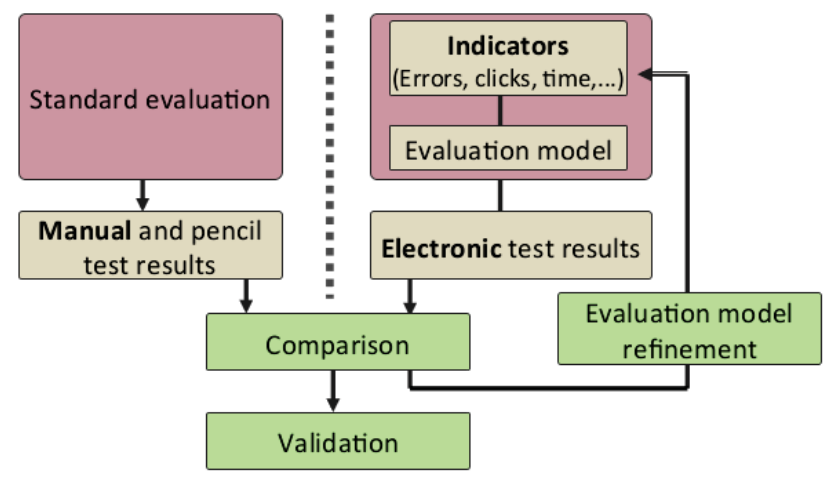

Figure 4: A view of task 1

pass the inclusion criteria, and then resulting as potentially affected by any neurological disorders. As shown in Figure 6 in the validation stage, the evaluation model will be refined to compute a score equivalent to standardized scores for pen and pencil test.

\section{CONCLUSIONS}

The SmartAgeing serious game is a novel methodology to detect cognitive impairments. Thanks to its accessibility features, it brings a friendly and fun way of testing elderly users. In addition, it brings an automatic way of reporting the users performance through the registration of many runtime parameters.

After the validation stage, we expect to be able to show its efficacy and ecological value. Once deployed for large-scale screening campaigns, it will require less resources both in terms of time and human activity that current methodologies. Finally, the SmartAgeing scenario can also be used to perform rehabilitation tasks for diagnosed patients. In this case, it will provide a coherent environment that will allow a continuous tracing of the patients evolution. We are currently working on this extension of the system.

\section{REFERENCES}

[1] D. Bavelier and R. Davidson. Games to do you good. Nature, 494:425-426, 2013.

[2] D. Bavelier, C. Green, D. Han, P. Renshaw, M. Merzenich, and D. Gentile. Brains on video games. Nature reviews Neuroscience, 12:763-768, 2012.

[3] E. Cherniack. Not just fun and games: applications of virtual reality in the identification and rehabilitation of cognitive disorders of the elderly. Disabil. Rehabil. Assist. Technol., 6(4), 2011.

[4] R. Darken and R. Durost. Mixed-dimension interaction in virtual environments. In ACM Symposium on Virtual Reality Software and Technology VRST '05, pages 38-45, 2005.

[5] MF. Folstein and SE. Folstein and PRG. Mc. Hugh. "Mini-mental state". A practical method for grading the cognitive state of patients for the clinician. Journal of Psychiatry Res.;12:189-198, 1975.
[6] B. Fernández-Calvo, R. Rodriguez-Pérez, L. Contador, A. Rubio-Santorum, and F. Ramos. Efficacy of cognitive training programs based on new software technologies in patients with Alzeimer-type dementia. Psicothema, 23(1):44-50, 2011.

[7] S. Kühn, T. Gleich, R. Lorenz, U. Lindenberger, and J. Gallinat. Playing Super Mario induces structural brain plasticity: gray matter changes resulting from training with a commercial video game. Molecular Psychiatry, 19:265-271, 2014.

[8] S. Moya, S.Grau, and D. Tost. The wise cursor: assisted selection in $3 \mathrm{D}$ serious games. The Visual Computer, 29(6-8):795-804, 2013

[9] S. Negash, G. Smith, S. Pankratz, J. Aakre, Y. Geda, R. Roberts, D. Knopman, B. Boeve, R. Ivnik, and R. Petersen. Successful aging: Definitions and prediction of longevity and conversion to mild cognitive impairment. Am. J. Geriatr. Psychiatry, 19(6):581-588, 2011.

[10] G. Optale, C. Urgesi, V. Busato, S. Marin, L. Piron, K. Priftis, L. Gamberini, S. Capodieci, and A. Bordin. Controlling memory impairment in elderly adults using virtual reality memory training: a randomized controlled pilot study. Neurorehabilitation and Neural Repair, 24(4):348-357, 2010.

[11] RC. Petersen RC, JC. Stevens, M. Ganguli et al. Practice parameter: early detection of dementia: mild cognitive impairment (an evidence-based review). Report of the Quality Standards Subcommittee of the American Academy of Neurology. Neurology, 56(9):1133-1142, 2001.

[12] G. Ravaglia, P. Forti, F. Montesi et al. Mild cognitive impairment: epidemiology and dementia risk in an elderly Italian population. J. American Geriatr. Soc., 56:51-58, 2008. 